\title{
Exact analytic Gorkov-Ginzburg-Landau theory of type-II superconductivity in the magneto-quantum oscillations limit
}

\author{
V. Zhuravlev ${ }^{1,2}$ and T. Maniv ${ }^{1, \text { * }}$ \\ ${ }^{1}$ Schulich Faculty of Chemistry, Technion-Israel Institute of Technology, Haifa 32000, Israel \\ ${ }^{2}$ Physics Department, Ort Braude College, P.O. Box 78, 21982 Karmiel, Israel
}

(Dated: November 24, 2018)

\begin{abstract}
A new Green's function representation is employed in a microscopic derivation of a GinzburgLandau theory of strongly type superconductivity at high magnetic fields. An exact analytical, physically transparent expression for the quartic term in the corresponding order parameter expansion is presented. The resulting expression reveals singular non-local contributions to the superconducting (SC) free energy, associated with highly coherent cyclotron motions of the paired electrons near the Fermi surface, which are strongly coupled to the vortex lattice. A major part of these contributions arises from incoherent scattering by the spatially averaged pair-potential, which is purely harmonic in the de Haas van Alphen frequency. However, coherent scatterings by the ordered vortex lattice generate, at low temperatures, large erratically oscillating (i.e. paramagnetic-diamagnetic) contribution to the SC free energy as a function of the magnetic field. Vortex lattice disorder, which tends to suppress this oscillatory component, is found to preserve the singular harmonic part of the $\mathrm{SC}$ free energy.

PACS numbers: $74.25 . \mathrm{Ha}, 74.25 . \mathrm{Uv}$
\end{abstract}

\section{INTRODUCTION}

Revealing the mechanism in which a clean, ordered superconducting (SC) material at very low temperatures responds to the application of an external magnetic field is of crucial importance for understanding the phenomenon of superconductivity at its most fundamental level. Surprisingly, as compared to high-field superconductivity under "non-ideal" conditions, i.e. in dirty or disordered materials at relatively high temperatures, the "ideal" SC state at low temperatures under high magnetic fields is currently not well understood even within the conventional BCS theory $\frac{1}{2}, 2$. Experimental detection of such high magnetic field states is currently restricted mainly to magneto-quantum oscillations techniques ${ }^{3}$, which provide researchers with detailed quantum information of the system investigated, but are difficult to analyze by the standard Fourier transform techniques due to the highly restricted range of magnetic fields available below the SC transition. There have been many reports on observation of de Haas van Alphen (dHvA) or Shubnikovde Haas $(\mathrm{SdH})$ oscillations in the SC states of strongly type-II superconductors, showing occasionally additional damping of the signal in the SC state with respect to the normal state signal (a partial list of references includes Refs $\underline{\underline{3}} \underline{\underline{-3}}$ ). However, their interpretation has not lead so far to any kind of consensus regarding the influence of the vortex matter on the oscillatory signal, partly because of the difficulties in the data analysis, and partly due to lack of a consistent theory with a quantitatively predictive power ${ }^{2}$. The mean-field theories based on a detailed exposition of the quasi-particle excitations obtained by solving the corresponding Bogoliubov-de Gennes (BdG) equations for an ordered vortex lattice $e^{\frac{9}{13}}$, provide insight into fine features of the Landau band structure, but lose their transparency very quickly and become heavily numerical at early stages of their application to any observable quantity. On the other hand, a simple formula for the additional damping ${ }^{14,15}$, used frequently in the literature for a manageable interpretation of experimental data, has been shown to be limited to situations of random vortex distributions ${ }^{2}$, and to the influence of the SC order parameter on the quasi particle relaxation rate $\frac{15}{5}$, neglecting important contributions to the oscillatory SC free energy.

Attempting to compare the results of the different theoretical approaches leads to great confusion. In the field range near $H_{c 2}$ where the $\mathrm{SC}$ order parameter is small and the leading (quadratic) term in the order parameter expansion of the SC free energy should be a good approximation 2 , the results of all numerical simulation of the BdG equations ${ }^{9}-13$ deviate markedly from this universal limiting value (see e.g. Fig. 8 in Ref ${ }^{13}$ ). The situation in the low magnetic fields region well bellow $H_{c 2}$ is even worse. Norman and MacDonald (NM) in their numerical simulations of the $\mathrm{BdG}$ equations reported 12 that the harmonic of the Fourier transform of the calculated magnetization in a finite interval of this regime varies erratically in sign and magnitude (see Fig.5 there) with no pattern that they could discern. Similar calculations carried out by Yasui and Kita ${ }^{13}$ have resulted in an additional damping rate which deviates drastically from both NM result and Maki's formula, showing erratically oscillating patterns of the corresponding Dingle plot, which seems to be indicative of some fundamental problem of numerical instability.

It is therefore desirable to derive an exact analytical expression for the SC thermodynamic potential which will enables one to identify the origin of this erratic behavior and carry out the calculation in a controlled fashion. In this paper we present such an exact analytical expression within the framework of the Gorkov-Ginzburg-Landau 
expansion of the free energy in the SC order parameter (or pair potential) up to fourth order. It is indeed found that highly correlated portions of the electronic cyclotron orbits involved in pairing have dominant contributions to the quartic and higher order terms of the SC free energy. The corresponding, singularly divergent distribution of the free energy obtained within an effective temporal representation, results in equally shared contributions from the spatially uniform component of the SC pair-potential and from its periodically modulated component associated with the vortex lattice.

The resulting expression of the free energy consists of two types: terms harmonic in the $\mathrm{dHvA}$ frequency and associated with the Landau level structure of the quasi particles, and erratically oscillating terms as functions of the magnetic field, associated with coherent scattering channels of the quasi particles by the vortex lattice. The latter are strongly enhanced when the length of a reciprocal vortex lattice vector coincides, or nearly coincides with the Fermi sphere diameter.

The resulting analytical expression also enables one to study the effect of disorder in the vortex lattice on the oscillatory free energy. In the white noise limit of the self-consistent Born approximation $(\mathrm{SCBA})^{15}$, the erratic oscillations associated with the coherent scattering by the vortex lattice are suppressed, yielding a result consistent with Maki's formula 14 . However, deviations from the SCBA should be carefully examined in light of the recent high-field low-temperature $\mu \mathrm{SR}$ measurements in the vortex-glass (peak-effect) region of borocarbide superconductors 16 , which have shown strong correlation betwen the enhanced additional damping of dHvA oscillations observed in the peak-effect region with enhanced vortex lattice disorder in this region 2 .

\section{GENERAL FORMULATION}

We consider a 2D strongly type-II (neglecting the effect of SC screening currents) superconductor in a perpendicular uniform magnetic field $\mathbf{H}=(0,0, H)$. Generalization to isotropic 3D systems is rather straightforward. It is assumed that the superconductor can be described by means of BCS-hamiltonian density for the usual singlet $s$-wave electron pairing

$$
\begin{aligned}
\mathcal{H}_{B C S} & =\Delta^{*}(\mathbf{r}) \psi(\mathbf{r}) \psi(\mathbf{r})+\Delta(\mathbf{r}) \psi^{*}(\mathbf{r}) \psi^{*}(\mathbf{r}) \\
& -\frac{1}{g_{B C S}}|\Delta(\mathbf{r})|^{2}
\end{aligned}
$$

where $\psi(\mathbf{r})$ is the single electron field operator and $g_{B C S}$ is BCS coupling constant (electron spin is neglected for the sake of simplicity). Within mean-field approximation the order parameter, $\Delta(\mathbf{r})=g_{B C S}\langle\psi(\mathbf{r}) \psi(\mathbf{r})\rangle$, should be determined self-consistently by minimizing the superconducting thermodynamic potential, $\Omega_{s c}(\Delta)$. We do not consider the problem in a fully self-consistent manner, assuming that the order parameter is described by a general vortex lattice state,

$$
\Delta(\mathbf{r})=\left(\frac{2 \pi}{a_{x}^{2}}\right)^{1 / 4} \Delta_{0} \varphi_{0}(\mathbf{r}) ;
$$

written in terms of a discrete set of ground-state Landau orbitals:

$$
\begin{aligned}
\varphi_{0}(x, y) & =e^{i x y} \sum_{n} e^{-i \theta n^{2}+i q_{n} x-\left(y+q_{n} / 2\right)^{2}} \\
& =e^{-\frac{1}{2}|z|^{2}+\frac{1}{2} z^{2}} \sum_{n} e^{i q_{n} z-\frac{q_{n}^{2}}{4}}
\end{aligned}
$$

where $z=x+i y$ and $q_{n}=\frac{2 \pi}{a_{x}} n=q_{0} n$ with the lattice spacing $a_{x}$ along the $x$ - axis and the angular parameter $\theta$ which determines the point symmetry of the vortex lattice. It is easy to see that for a general (rhombic) vortexlattice geometry, determined by the angular parameter $\theta, a_{x}^{2}=\pi / \sqrt{1-(\theta / \pi)^{2}}$. For the Abrikosov triangular lattice: $\theta=\pi / 2$ and $q_{0}=\frac{2 \pi}{a_{x}}=3^{1 / 4} \sqrt{2 \pi}$.

We use the symmetric gauge with vector potential $\mathbf{A}=\frac{1}{2}[\mathbf{H} \times \mathbf{r}]=\frac{1}{2} H(-y, x, 0)$ and dimensionless space coordinates measured in units of the electronic magnetic length, $a_{H}=\sqrt{c \hbar / e H}$. The amplitude of the order parameter, $\Delta_{0}^{2}=S^{-1} \int d^{2} \mathbf{r}_{i}\left|\Delta\left(\mathbf{r}_{i}\right)\right|^{2}$, where $S=\pi N$ is the area of the $2 \mathrm{D}$ superconductor and $N$ is the number of vortices, is treated as a variational parameter for minimizing $\Omega_{s c}(\Delta)$.

The thermodynamic potential, $\Omega_{s c}(\Delta)$, can be written as a Taylor expansion in the $\mathrm{SC}$ order parameter $\underline{\underline{1}}$ :

$$
\begin{aligned}
\Omega_{s c}\left(\Delta_{0}\right) & =S \frac{\Delta_{0}^{2}}{\left(\hbar \omega_{c}\right)^{2} g_{B C S}}+\sum_{n=1} \frac{(-1)^{n}}{n} \Omega_{2 n}\left(\Delta_{0}\right), \\
\Omega_{2 n} & =\Omega_{2 n}^{(0)} \int d^{2}\{\mathbf{r}\} \widetilde{\Gamma}_{2 n}(\{\mathbf{r}\}) \widetilde{K}_{2 n}(\{\mathbf{r}\}), \\
\Omega_{2 n}^{(0)} & =\left(\frac{2 \pi}{a_{x}^{2}}\right)^{2 n / 4} \frac{1}{(2 \pi)^{2 n}} k_{B} T a_{H}^{2}\left|\frac{\Delta_{0}}{\hbar \omega_{c}}\right|^{2 n} .
\end{aligned}
$$

where:

$$
\begin{aligned}
& \widetilde{K}_{2 n}(\{\mathbf{r}\})=\left(2 \pi \hbar^{2} / m\right)^{2 n} \sum_{\nu} G_{0}^{*}\left(\mathbf{r}_{1}, \mathbf{r}_{2}, \omega_{\nu}\right) \times \\
& G_{0}\left(\mathbf{r}_{2}, \mathbf{r}_{3}, \omega_{\nu}\right) \ldots G_{0}^{*}\left(\mathbf{r}_{2 n-1}, \mathbf{r}_{2 n}, \omega_{\nu}\right) G_{0}\left(\mathbf{r}_{2 n}, \mathbf{r}_{1}, \omega_{\nu}\right) \\
& \widetilde{\Gamma}_{2 n}(\{\mathbf{r}\})=g^{*}\left(\mathbf{r}_{1}, \mathbf{r}_{2}\right) g\left(\mathbf{r}_{2}, \mathbf{r}_{3}\right) \ldots g^{*}\left(\mathbf{r}_{2 n-1}, \mathbf{r}_{2 n}\right) \times( \\
& g\left(\mathbf{r}_{2 n}, \mathbf{r}_{1}\right) \varphi_{0}\left(\mathbf{r}_{1}\right) \varphi_{0}^{*}\left(\mathbf{r}_{2}\right) \ldots \varphi_{0}\left(\mathbf{r}_{2 n-1}\right) \varphi_{0}^{*}\left(\mathbf{r}_{2 n}\right)
\end{aligned}
$$

Here we use the normal state single electron Green's function in the uniform magnetic field, which is given by the well known expression, $G\left(\mathbf{r}_{2}, \mathbf{r}_{1}, \omega_{\nu}\right)=$ $g\left(\mathbf{r}_{2}, \mathbf{r}_{1}\right) G_{0}\left(\mathbf{r}_{2}, \mathbf{r}_{1}, \omega_{\nu}\right)$, where:

$$
G_{0}\left(\mathbf{r}_{2}, \mathbf{r}_{1}, \omega_{\nu}\right) \equiv G_{0}\left(\rho, \omega_{\nu}\right)=\frac{1}{2 \pi a_{H}^{2}} \sum_{n} \frac{e^{-\rho^{2} / 4} L_{n}\left(\rho^{2} / 2\right)}{\mu_{F}-\varepsilon_{n}+i \hbar \omega_{\nu}}
$$


$\rho=\mathbf{r}_{\mathbf{2}}-\mathbf{r}_{\mathbf{1}}$, and $g\left(\mathbf{r}_{2}, \mathbf{r}_{1}\right)=e^{-\frac{i}{2}\left[\mathbf{r}_{2} \times \mathbf{r}_{1}\right]}$ is the usual gauge factor. Also note that in the above equations $\omega_{\nu}=\pi k_{B} T(2 \nu+1) / \hbar, \nu=0, \pm 1, \ldots$ is the Matsubara frequency at temperature $T, \mu_{F}$ is the chemical potential, and $\varepsilon_{n}=\hbar \omega_{c}(n+1 / 2), n=0,1,2, \ldots$ is a Landau level energy with $\omega_{c}=e H / m c$ the cyclotron frequency.

It will be very helpful to use an equivalent representation of the translational invariant part of the Green's function for $\omega_{\nu}>0$,i.e.:

$$
\begin{aligned}
G_{0}(\rho) & =\frac{1}{2 \pi \hbar \omega_{c}} \int_{0}^{\infty} d \tau e^{i \tau\left[n_{F}+i \varpi_{\nu}\right]} \frac{\exp \left(-\frac{\rho^{2}}{4} \frac{1+e^{-i \tau}}{1-e^{-i \tau}}\right)}{1-e^{-i \tau}} \\
& =\frac{1}{2 \pi \hbar \omega_{c}} \int_{0}^{\infty} \frac{d \tau}{\alpha} e^{i \tau\left[n_{F}+i \varpi_{\nu}\right]-\mu \rho^{2}}
\end{aligned}
$$

which can be easily derived from Eq 5 by using the integral representation of $\left(\mu_{F}-\varepsilon_{n}+i \hbar \omega_{\nu}\right)^{-1}$ and the generating function of the Laguerre polynomials. The resulting expression is written in terms of the following dimensionless quantities: $\alpha \equiv 1-e^{-i \tau}, \quad \mu \equiv \frac{1}{4} \frac{1+e^{-i \tau}}{1-e^{-i \tau}}$, $\left(\mu_{F}-\varepsilon_{n}\right)=\hbar \omega_{c}\left(n_{F}-n\right)$ and $\omega_{\nu}=\hbar \omega_{c} \varpi_{\nu}$. Note also that for $\varpi_{\nu}<0, \tau$ in Eq $[$ should be replaced with $-\tau$, yielding the complex conjugate of the expression for $\varpi_{\nu}>0$.

Exploiting the integral representation, Eq 6. we can rewrite the electronic kernel $\widetilde{K}_{2 n}(\{\mathbf{r}\})$ in the form:

$$
\begin{aligned}
& \widetilde{K}_{2 n}(\{\mathbf{r}\})=\prod_{j} \int_{0}^{\infty} d \tau_{j} e^{-i \varepsilon_{j} \tau_{j} n_{F}-\varpi_{\nu} \tau_{j}} \frac{1}{\alpha_{j}} \exp \left(-\mu_{j} \rho_{j}^{2}\right) \\
& \varepsilon_{j}=(-1)^{j+1}, \quad \alpha_{j}=1-e^{i \varepsilon_{j} \tau_{j}}, \quad \mu_{j}=\frac{1}{4} \frac{1+e^{i \varepsilon_{j} \tau_{j}}}{1-e^{i \varepsilon_{j} \tau_{j}}}
\end{aligned}
$$

This representation of $\Omega_{s c}\left(\Delta_{0}\right)$ has an obvious advantage over the original expression: all space integrals are of Gaussian forms and, therefore, can be calculated analytically. Unfortunately, gauge factors mix all electron coordinates so that the calculation of the higher order terms is not trivial.

\section{QUARTIC VERSUS QUADRATIC TERMS: EFFECT OF THE VORTEX LATTICE}

\section{A. The quadratic term}

The second order term have been calculated long ago. We repeat the calculation to illustrate the advantage of using the Green's function in the special representation, Eq, 6 .

The vertex part in Eq, 4 can be written as

$$
\begin{aligned}
\widetilde{\Gamma}_{2}\left(\mathbf{r}_{1}, \mathbf{r}_{2}\right) & =g^{*}\left(\mathbf{r}_{1}, \mathbf{r}_{2}\right) g\left(\mathbf{r}_{2}, \mathbf{r}_{1}\right) \varphi_{0}\left(\mathbf{r}_{1}\right) \varphi_{0}^{*}\left(\mathbf{r}_{2}\right) \\
& =\sum_{n, m=-\infty}^{\infty} e^{\zeta_{n m}^{(2)}}
\end{aligned}
$$

where $\zeta_{n m}^{(2)}=i\left(x_{1} y_{2}-y_{1} x_{2}\right)+i x_{1} y_{1}-i x_{2} y_{2}+$ $i q_{n} x_{1}-\left(y_{1}+q_{n} / 2\right)^{2}-i q_{m} x_{2}-\left(y_{2}+q_{m} / 2\right)^{2}$. Noting that the dependence on the center of mass coordinates, $\quad \mathbf{R} \equiv \frac{\mathbf{r}_{1}+\mathbf{r}_{2}}{2}$, appears only in the vertex part, one can extract this dependence from $\zeta_{n m}^{(2)}$, ending with two integrals over the center of mass: $\int d R_{x} \exp \left[i\left(q_{n} R_{x}-q_{m} R_{x}\right)\right]=a_{x} N_{x} \delta_{n m}$ and $\int d R_{y}^{\prime} \exp \left(-2 R_{y}^{\prime 2}\right)=\sqrt{\frac{\pi}{2}}$. Here $N_{x}$ is the number of vortices along the $x$-direction and $R_{y}^{\prime}$ is a shifted $R_{y}$ coordinate. The remaining function, $\zeta_{n m}^{(2)} \rightarrow-\frac{1}{2} \rho^{2}$ does not depend on $n$, so that summation over $n$ gives the number of Landau orbitals along the $y$-axis, $N_{y}$. Consequently, the quadratic term can be written as

$$
\begin{aligned}
\Omega_{2 \nu}= & a_{x} \sqrt{\frac{\pi}{2}} N_{x} N_{y} \Omega_{2}^{(0)} \int_{0}^{\infty} \int_{0}^{\infty} d \tau_{1} d \tau_{2} \\
& e^{i n_{F}\left(\tau_{2}-\tau_{1}\right)-\varpi_{\nu}\left(\tau_{1}+\tau_{2}\right)} \times \\
& \frac{1}{\alpha_{1} \alpha_{2}} \int d^{2} \rho \exp \left(-\mu_{1} \rho^{2}-\mu_{2} \rho^{2}-\frac{1}{2} \rho^{2}\right)
\end{aligned}
$$

or, after integrating over relative coordinates, $\rho$, as

$$
\begin{aligned}
\Omega_{2 \nu}= & a_{x} \sqrt{\frac{\pi}{2}} N \Omega_{2}^{(0)} \int_{0}^{\infty} \int_{0}^{\infty} d \tau_{1} d \tau_{2} \\
& e^{i n_{F}\left(\tau_{2}-\tau_{1}\right)-\varpi_{\nu}\left(\tau_{1}+\tau_{2}\right)} \frac{1}{\alpha_{1}+\alpha_{2}}
\end{aligned}
$$

where $N=N_{x} N_{y}$ is a number of vortices in the system.

The dominant contributions to the $\tau$-integrals originates in the poles of the integrand where $\alpha_{1,2} \rightarrow 0$, namely at $\tau_{j} \rightarrow 2 n_{j} \pi, n_{j}=0, \pm 1, .$. , where the first exponent $i n_{F}\left(\tau_{2}-\tau_{1}\right)$ is equal to $2 i \pi n_{F} n, n=0, \pm 1, \ldots$, corresponding to exact harmonics of the $\mathrm{dHvA}$ frequency $F=n_{F} H$. We therefore conclude that the quadratic term is dominated by harmonics of the $\mathrm{dHvA}$ frequency which implies that to leading order in the GGL expansion the Landau levels structure is not distorted by the vortex lattice. This result is consistent with the well known property of the quadratic term to be independent of the vortex lattice structure.

Considering the first harmonic for the sake of illustration, we shift $\tau_{2} \rightarrow 2 \pi+\tau_{2}$ and expand $\alpha_{1}+\alpha_{2}$ in $\tau_{1}$ and $\tau_{2}$ for $\tau_{1} \ll 1$ and $\left|\tau_{2}\right| \ll 1: \alpha_{1}+\alpha_{2} \simeq$ $i\left(\tau_{2}-\tau_{1}\right)+\frac{1}{4}\left(\tau_{1}+\tau_{2}\right)^{2}$. Here the term $\frac{1}{4}\left(\tau_{1}-\tau_{2}\right)^{2}$ was neglected since $\left(\tau_{2}-\tau_{1}\right) \sim\left(\tau_{1}+\tau_{2}\right)^{2}$. Noting, further, that if $\tau_{1}+\tau_{2}<0$ the pole is located out of the integration interval, and calculating the corresponding Cauchy integral over the $\left(\tau_{2}-\tau_{1}\right)$-variable for $\tau_{1}+\tau_{2} \geq 0$, one obtains: $\frac{1}{2} k_{B} T a_{H}^{2} N \frac{\pi^{3 / 2}}{\sqrt{n_{F}}}\left(\frac{\Delta_{0}}{\hbar \omega_{c}}\right)^{2} e^{2 i \pi n_{F}-2 \pi \varpi_{\nu}}$. A similar expression can be derived by expanding near the symmetric point $\tau_{1} \rightarrow 2 \pi+\tau_{1}$ and $\tau_{2} \rightarrow \tau_{2}$ with $\left|\tau_{1}\right| \ll 1$ and $\tau_{2} \ll 1$. Therefore, the quadratic term is written as

$$
\Omega_{2}^{(1 h)}=k_{B} T a_{H}^{2} N \frac{\pi^{3 / 2}}{\sqrt{n_{F}}}\left(\frac{\Delta_{0}}{\hbar \omega_{c}}\right)^{2} R e e^{2 i \pi n_{F}-2 \pi \varpi_{\nu}}
$$

Since near the poles $\mu_{j} \sim \frac{1}{\tau_{j}} \gg 1$, the spatial integral in Eq,7 is dominated by very small distances, a result 
consistent with the locality of the quadratic term. Also note that the final expression does not depend on $a_{x}$, a result consistent with the fact that the structure of the vortex lattice does not influence the quadratic term.

\section{B. The quartic term}

\section{Useful analytical expressions}

The calculation of the next order term, the quartic term, is much more complicated since, unlike the quadratic term, it is strongly affected by the coupling of the electrons to the vortex lattice. However, the use of the representation, Eq, 6 for the single electron Green's functions facilitates greatly the entire 8-fold spatial integration by transforming the corresponding integrand into a multiple Gaussian form. Following the derivation described in detail in Appendix A the quartic term can be written as a 4D 'temporal' integral:

$$
\begin{aligned}
\Omega_{4 \nu}= & \Omega_{4}^{(0)} L_{x} N_{y} \frac{\sqrt{\pi}}{2}\left|\operatorname{det} M^{-1}\right|^{2} \int_{0}^{\infty} d \tau_{1} d \tau_{2} d \tau_{3} d \tau_{4} \\
& e^{-\varpi_{\nu}\left(\tau_{1}+\tau_{2}+\tau_{3}+\tau_{4}\right)-i n_{F}\left(\tau_{1}-\tau_{2}+\tau_{3}-\tau_{4}\right)} \Phi[\tau] L[\tau]
\end{aligned}
$$

where

$$
\begin{aligned}
\Phi[\tau] & =\frac{1}{\alpha_{1} \alpha_{2} \alpha_{3} \alpha_{4}} \frac{\pi}{\beta_{0}} \frac{\pi^{2}}{\operatorname{det} U} \\
L[\tau] & =\sum_{s t} \exp \left[-\frac{1}{4} q_{0}^{2}\left(s^{2}+t^{2}\right)+\frac{1}{4} L^{T} U^{-1} L\right]
\end{aligned}
$$

where the vector $L$ is given in Eq A5, and the matrix $U$ in EqA6

The calculation of $\operatorname{det} U$ can be done by using the relations $\mu_{i}+\frac{1}{4}=\frac{1}{2 \alpha_{i}}$, and noting that it can be factorized to: $\operatorname{det} U=\lambda_{b} \lambda_{a}$ where $\lambda_{a}=\frac{1}{2 \beta}\left(\lambda_{3}-\lambda_{2}\right), \quad \lambda_{b}=$ $\frac{1}{2 \beta}\left(\lambda_{3}+\lambda_{2}\right)$ with $\beta=\frac{1}{2}\left(\frac{1}{\alpha_{1}}+\frac{1}{\alpha_{2}}+\frac{1}{\alpha_{3}}+\frac{1}{\alpha_{4}}\right), \lambda_{3}=$ $\frac{1}{\alpha_{1} \alpha_{2} \alpha_{3}}+\frac{1}{\alpha_{2} \alpha_{3} \alpha_{4}}+\frac{1}{\alpha_{1} \alpha_{2} \alpha_{4}}+\frac{1}{\alpha_{1} \alpha_{3} \alpha_{4}}, \quad \lambda_{2}=\frac{1}{\alpha_{1} \alpha_{3}}-\frac{1}{\alpha_{2} \alpha_{4}}$. Substituting these values to $\Phi[\tau]$ one arrives at the compact expression:

$$
\Phi[\tau]=\frac{2 \pi^{3}}{\alpha_{1}+\alpha_{2}+\alpha_{3}+\alpha_{4}} \frac{1}{\left(1-\gamma^{2}\right)^{1 / 2}}
$$

with

$$
\gamma=\frac{\lambda_{2}}{\lambda_{3}}=\frac{\alpha_{2} \alpha_{4}-\alpha_{1} \alpha_{3}}{\alpha_{1}+\alpha_{2}+\alpha_{3}+\alpha_{4}} .
$$

The calculation of the exponential term results in

$$
\begin{aligned}
\frac{1}{4} L^{T} \bar{U}^{-1} L & =-q_{0}^{2}\left[\frac{\lambda_{a}-\lambda_{b}}{\lambda_{b}} s^{2}+\frac{\lambda_{b}-\lambda_{a}}{\lambda_{a}} t^{2}\right] \\
& =2 q_{0}^{2} \gamma\left(\frac{s^{2}}{\gamma+1}+\frac{t^{2}}{\gamma-1}\right)
\end{aligned}
$$

Substituting Eqs. 12 and 14 to Eq. 9 we obtain the final result

$$
\begin{aligned}
\Omega_{4 \nu}= & \frac{1}{2} k_{B} T a_{H}^{2} N\left|\frac{\Delta_{0}}{\hbar \omega_{c}}\right|^{4} I_{4} \\
I_{4}= & \int_{0}^{\infty} d \tau_{1} d \tau_{2} d \tau_{3} d \tau_{4} \\
& e^{-\varpi_{\nu}\left(\tau_{1}+\tau_{2}+\tau_{3}+\tau_{4}\right)-i n_{F}\left(\tau_{1}-\tau_{2}+\tau_{3}-\tau_{4}\right)} \times \\
& \frac{\beta(\gamma)}{\alpha_{1}+\alpha_{2}+\alpha_{3}+\alpha_{4}}
\end{aligned}
$$

where:

$$
\begin{aligned}
\beta(\gamma)= & \frac{\sqrt{\pi}}{a_{x}} \frac{1}{\left(1-\gamma^{2}\right)^{1 / 2}} \times \\
& \sum_{s t} \exp \left[-2 i \theta s t-\frac{1}{4} q_{0}^{2}\left(\frac{1-\gamma}{1+\gamma} s^{2}+\frac{1+\gamma}{1-\gamma} t^{2}\right)\right]
\end{aligned}
$$

\section{Major analytical properties}

The structure function $\beta(\gamma)$, expressed in Eq,16, controls the coupling between the four electrons involved and the vortex lattice. Its most remarkable feature is associated with the dual singular points at $\gamma \rightarrow \pm 1$, where the lattice sums over $s$ or $t$ (depending on whether $1-\gamma \rightarrow 0$ or $1+\gamma \rightarrow 0$, respectively) can be replaced by integrals (over $\widetilde{s}=s \sqrt{(1-\gamma) / 2}$ or $\tilde{t}=t \sqrt{(1+\gamma) / 2}$, respectively), enhancing the singularities of the corresponding pre-exponential factors to simple poles:

$$
\begin{aligned}
& \beta(\gamma \rightarrow 1) \rightarrow \frac{\sqrt{\pi}}{a_{x}} \frac{1}{(1-\gamma)} \int d \widetilde{s} e^{-\frac{1}{4} q_{0}^{2} \widetilde{s}^{2}} \times \\
& \sum_{t} \exp \left[-\frac{1}{2} q_{0}^{2}\left(\frac{1}{1-\gamma}\right) t^{2}-i \sqrt{2} \theta \widetilde{s}\left(\frac{1}{1-\gamma}\right)^{1 / 2} t\right] \\
& \rightarrow \frac{1}{(1-\gamma)}, \\
& \beta(\gamma \rightarrow-1) \rightarrow \frac{\sqrt{\pi}}{a_{x}} \frac{1}{(1+\gamma)} \int d \tilde{t} e^{-\frac{1}{4} q_{0}^{2} \widetilde{t}^{2} \times} \\
& \sum_{s} \exp \left[-\frac{1}{2} q_{0}^{2}\left(\frac{1}{1+\gamma}\right) s^{2}-i \sqrt{2} \theta \tilde{t}\left(\frac{1}{1+\gamma}\right)^{1 / 2} s\right] \\
& \rightarrow \frac{1}{(1+\gamma)}
\end{aligned}
$$

Note that in the sum over the remaining variable, $t$ or $s$, only the single term $t$ (or $s)=0$ survives, due to the large negative real-part values of the corresponding exponent.

It is interesting to note that the values of the individual electronic "time" variables, $\tau_{j}$, satisfying the singular conditions, $\gamma \rightarrow \pm 1$, are given, respectively, by:

$$
\tau_{1}=\tau_{3} \rightarrow 0, \tau_{2} \rightarrow n \pi-\tau, \tau_{4} \rightarrow n \pi+\tau
$$


or

$$
\tau_{1} \rightarrow n \pi-\tau, \tau_{3} \rightarrow n \pi+\tau, \tau_{2}=\tau_{4} \rightarrow 0
$$

where $\tau$ is an arbitrary real number in the interval: $-\pi \leq \tau \leq \pi$, and $n=0, \pm 1, \pm 2, \ldots$. Thus, the electrons at such highly correlated pairs of cyclotron orbits are resonantly coupled to the entire vortex lattice, yielding only purely harmonic contributions to the SC free energy in the dHvA frequency $F=n_{F} H$ since under these conditions: $e^{-i n_{F}\left(\tau_{1}-\tau_{2}+\tau_{3}-\tau_{4}\right)} \rightarrow e^{-2 \pi i n n_{F}}$. Note also that at the singular points the factor $e^{-\varpi_{\nu}\left(\tau_{1}+\tau_{2}+\tau_{3}+\tau_{4}\right)}$ is equal to $e^{-2 \pi\left|n \varpi_{\nu}\right|}$, determining the thermal damping of the quantum oscillatory part of the SC free energy, and a natural (thermal) cutoff for the integrals over $\tau_{j}$.

Another type of singularities of the integrand in Eq 15 corresponds to the vanishing denominator $\alpha_{1}+\alpha_{2}+\alpha_{3}+$ $\alpha_{4}$, which takes place at simultaneous vanishing of all $\alpha_{j}=1-e^{i \varepsilon_{j} \tau_{j}}$, namely when $\tau_{j} \rightarrow 2 \pi n_{j}, n_{j}=0,1,2, \ldots$ At the corresponding poles the effective coupling parameter of the electrons to the vortex lattice $\gamma \rightarrow 0$, and one recovers the well known local approximation in which the electrons are only weakly coupled to the vortex lattice.

\section{Effect of the vortex lattice}

The effect of the vortex lattice on the free energy can be expressed more clearly by transforming the lattice double sum in Eq16 into a 2D reciprocal vortex lattice summation. To do so the summation over $t$ is transformed by means of Poisson formula into:

$$
\begin{aligned}
& \sum_{m=-\infty}^{\infty} \int_{-\infty}^{\infty} d t \exp \left[2 i(\pi m-\theta s) t-\left(\frac{\pi}{a_{x}}\right)^{2}\left(\frac{1+\gamma}{1-\gamma}\right) t^{2}\right] \\
= & \sqrt{\frac{a_{x}^{2}}{\pi}}\left(\frac{1-\gamma}{1+\gamma}\right)^{1 / 2} \times \\
& \sum_{m=-\infty}^{\infty} \exp \left\{-\left(\frac{1-\gamma}{1+\gamma}\right)(\pi m-\theta s)^{2}\left(\frac{a_{x}}{\pi}\right)^{2}\right\}
\end{aligned}
$$

, so that:

$$
\begin{aligned}
& \beta(\gamma)=\frac{1}{(1+\gamma)} \sum_{s, m=-\infty}^{\infty} \\
& \exp \left\{-\left(\frac{1-\gamma}{1+\gamma}\right)\left[\left(\frac{\pi}{a_{x}}\right)^{2} s^{2}+(\pi m-\theta s)^{2}\left(\frac{a_{x}}{\pi}\right)^{2}\right]\right\}
\end{aligned}
$$

Now, using two primitive vectors spanning the vortex lattice: $\mathbf{a}=\widehat{x} a_{x}, \mathbf{b}=\widehat{x} b_{x}+\widehat{y} b_{y}$, with $b_{y}=\pi / a_{x}$, the corresponding primitive vectors spanning the reciprocal vortex lattice are: $\mathbf{a}^{*}=\widehat{x} b_{y}-\widehat{y} b_{x}, \mathbf{b}^{*}=\widehat{y} a_{x}$, so that

$$
\left(\frac{\pi}{a_{x}}\right)^{2} s^{2}+(\theta s-\pi m)^{2}\left(\frac{a_{x}}{\pi}\right)^{2}=\left(s \mathbf{a}^{*}+m \mathbf{b}^{*}\right)^{2}
$$

and

$$
\begin{aligned}
\beta(\gamma) & =\frac{1}{(1+\gamma)} \sum_{s, m=-\infty}^{\infty} \exp \left[-\left(\frac{1-\gamma}{1+\gamma}\right)\left|\mathbf{G}_{s m}\right|^{2}\right] \\
\mathbf{G}_{s m} & \equiv s \mathbf{a}^{*}+m \mathbf{b}^{*}
\end{aligned}
$$

A similar procedure in which Poisson formula is used with respect to the summation over $s$ leads to an expression identical to Eq20 after exchanging $\gamma \longleftrightarrow-\gamma$. Since under the integrations over all $\tau_{j}, j=1, \ldots, 4$, a given value of $\gamma$ (corresponding to a given selection of $\left.\tau_{1}, \tau_{3}, \tau_{2}, \tau_{4}\right)$ always appears with its opposite value $-\gamma$ (corresponding to the values of $\tau_{1}, \tau_{3}$ exchanged with those of $\left.\tau_{2}, \tau_{4}\right)$ one can always replace $\beta(\gamma)$ in Eq[15] with the symmetric expression:

$$
\begin{aligned}
& \beta^{\text {sym }}(\gamma)=\sum_{\mathbf{G}} \beta_{\mathbf{G}}^{\text {sym }}(\gamma)= \\
& \frac{1}{2} \sum_{\mathbf{G}}\left\{\frac{1}{(1+\gamma)} \exp \left[-\left(\frac{1-\gamma}{1+\gamma}\right)|\mathbf{G}|^{2}\right]\right. \\
& \left.+\frac{1}{(1-\gamma)} \exp \left[-\left(\frac{1+\gamma}{1-\gamma}\right)|\mathbf{G}|^{2}\right]\right\}
\end{aligned}
$$

without altering the result of $I_{4}$.

Near the singular points $\gamma= \pm 1 \mathrm{Eq} 21$ describes two additive coherent processes of two electron pairs moving in cyclotron orbits on the Fermi surface and undergoing scatterings by the vortex lattice. Near the singular point $\gamma \rightarrow 1$, where the positions of the electrons labeled $(1,3)$ along their cyclotron orbit coincide (i.e. for $\tau_{1}=\tau_{3} \rightarrow 0$, see Eq 17), the other two electrons, labeled $(2,4)$, are moving coherently along their cyclotron orbits in opposite directions (i.e. $\tau_{2} \rightarrow n \pi-\tau, \tau_{4} \rightarrow n \pi+\tau$, see Eq[18). Thus, the singular $\gamma \rightarrow 1$ contribution of the first term within the brackets in Eqs 21 is associated with the electrons labeled $(1,3)$ and involves many $G$-vectors, whereas the singular $\gamma \rightarrow 1$ contribution of the second term is associated with the other two electrons labeled $(2,4)$ and involves only the $G=0$ channel. Similarly, near the dual singular point $\gamma \rightarrow-1$, where the $(1,3)$ electrons are moving in opposite directions (i.e. $\left.\tau_{1} \rightarrow n \pi-\tau, \tau_{3} \rightarrow n \pi+\tau\right)$ and the positions of the $(2,4)$ electrons along their orbit coincide (i.e. $\tau_{2}=\tau_{4} \rightarrow 0$ ), the contribution of the first term involves only the $G=0$ channel, whereas the contribution of the second term involves many $G$-vectors.

The physical meaning of the singular $\gamma \rightarrow \pm 1$ contributions is therefore apparent: The two electrons whose positions on the cyclotron orbit coincide at the singular point undergo local mutual scattering and so exchange many $G$-vectors through the vortex lattice during the scattering process, while those electrons moving coherently on a large cyclotron orbit in opposite directions are mutually scattered through the entire vortex lattice, and so do not exchange momentum.

The resulting leading contributions to $\beta^{\text {sym }}(\gamma)$ can be therefore written in terms of very simple formulas: The 
forward scattering contribution takes the form:

$$
\beta_{\mathbf{G}=\mathbf{0}}^{s y m}(\gamma)=\frac{1}{2}\left(\frac{1}{1+\gamma}+\frac{1}{1-\gamma}\right)
$$

whereas the rest of the reciprocal lattice contributions, $\sum_{\mathbf{G} \neq \mathbf{0}} \beta_{\mathbf{G}}^{\text {sym }}(\gamma)$, which involve increasingly large numbers of reciprocal lattice vectors as $\gamma \rightarrow \pm 1$, can be well approximated in these limiting cases by the two-dimensional integral: $\int \beta_{\mathbf{G}}^{\text {sym }}(\gamma) d^{2} G$, yielding:

$$
\sum_{\mathbf{G} \neq \mathbf{0}} \beta_{\mathbf{G}}^{\text {sym }}(\gamma) \rightarrow \int \beta_{\mathbf{G}}^{\text {sym }}(\gamma) d^{2} G=\frac{1}{2}\left\{\begin{array}{cc}
\frac{1}{1-\gamma}, \gamma \rightarrow 1 \\
\frac{1}{1+\gamma}, \gamma \rightarrow-1
\end{array}\right\}
$$

Note that the $G=0$ term, given by Eq. 22, represents the effect of the spatially uniform component of the SC order parameter on the free energy whereas the rest of the terms in Eq. 21 correspond to all possible umklapp (coherent) scattering processes by the vortex lattice.

To gain further insight into this remarkable coupling to the vortex lattice we may expand $\beta(\gamma) /\left(\alpha_{1}+\alpha_{2}+\alpha_{3}+\alpha_{4}\right)$ about one of the singular points, say $\gamma=1$, and carry out the $\tau_{j^{-}}$ integrations to derive a more transparent (but approximate) expression for $I_{4}$. Focusing, for simplicity, on the first harmonic of the dHvA frequency $F=n_{F} H$, our small expansion parameters are (see also Sec.B4): $\widetilde{\xi}_{1}=\frac{1}{4}\left(\tau_{1}+\tau_{2}+\tau_{3}+\tau_{4}\right)-\pi / 2, \widetilde{\xi}_{2}=$ $\frac{1}{2}\left(\tau_{1}-\tau_{2}+\tau_{3}-\tau_{4}\right)+\pi, \widetilde{\xi}_{3}=\tau_{1}-\tau_{3}$, and $\widetilde{\xi}_{4}=\tau_{4}-\tau_{2}$, so that to second order, the key composite variables are given by:

$$
\frac{1-\gamma}{1+\gamma} \simeq-\frac{1}{4} i \widetilde{\xi}_{2}+\frac{1}{16}\left(4 \widetilde{\xi}_{1}^{2}+\widetilde{\xi}_{3}^{2}\right)
$$

and:

$$
\begin{aligned}
I_{4} \rightarrow & e^{2 \pi i n_{F}} e^{-2 \pi \varpi_{\nu}} \int_{0}^{\infty} d \widetilde{\xi}_{1} e^{-4 \varpi_{\nu} \widetilde{\xi}_{1}} \times \\
& \sum_{\mathbf{G}} \int_{-2 \widetilde{\xi}_{1}}^{2 \widetilde{\xi}_{1}} d \widetilde{\xi}_{2} \exp \left\{i \widetilde{\xi}_{2}\left[\frac{1}{4}|\mathbf{G}|^{2}-2 n_{F}\right]\right\} \times \\
& \int_{-\left(2 \widetilde{\xi}_{1}+\widetilde{\xi}_{2}\right)}^{2 \widetilde{\xi}_{1}+\widetilde{\xi}_{2}} d \widetilde{\xi}_{3} \exp \left\{-\left(\widetilde{\xi}_{1}^{2}+\frac{1}{4} \widetilde{\xi}_{3}^{2}\right) \frac{1}{4}|\mathbf{G}|^{2}\right\} \times \\
& \int_{-\left(2 \widetilde{\xi}_{1}-\widetilde{\xi}_{2}\right)}^{2 \widetilde{\xi}_{1}-\widetilde{\xi}_{2}} d \widetilde{\xi}_{4}
\end{aligned}
$$

Considering the umklapp scattering terms with large vectors $\mathbf{G}$ it is clear that the dominant contributions originate from reciprocal lattice vectors satisfying: $\frac{1}{2}|\mathbf{G}| \approx \sqrt{2 n_{F}}$, namely having length close to the Fermi surface diameter. Furthermore, due to the large values of $n_{F}$ and the discrete nature of $\mathbf{G}$ (which are measured in units of the magnetic length) with an elementary unit of about $\pi$, the integration over $\xi_{2}$ yields erratically oscillating function of $n_{F}$, which reflects dramatic influence of the vortex lattice on the fermionic quasi-particles at high magnetic field.

\section{Numerical calculations}

For numerical calculations we use Eq 15 assuming a square vortex lattice with $a_{x}=\sqrt{\pi}$. Performing Poisson summation over $s$ or $t$ in Eq 16 one can transform $\beta(\gamma)$ into simpler, equivalent forms:

$$
\begin{aligned}
\beta_{s q}(\gamma) & =\frac{1}{1-\gamma} \sum_{m t} \exp \left[-\pi \frac{1+\gamma}{1-\gamma}\left(m^{2}+t^{2}\right)\right] \\
& =\frac{1}{1-\gamma}\left(\sum_{n} \exp \left[-\pi \frac{1+\gamma}{1-\gamma} n^{2}\right]\right)^{2} \\
& =\frac{1}{1+\gamma}\left(\sum_{n} \exp \left[-\pi \frac{1-\gamma}{1+\gamma} n^{2}\right]\right)^{2}
\end{aligned}
$$

The integrals in Eq 15 can be more conveniently evaluated by transforming to the new variables (shifted with respect to $\widetilde{\xi}_{i}$, defined above Eq 24): $\xi_{1}=$ $\frac{1}{4}\left(\tau_{1}+\tau_{2}+\tau_{3}+\tau_{4}\right), \xi_{2}=\frac{1}{2}\left(\tau_{1}-\tau_{2}+\tau_{3}-\tau_{4}\right), \xi_{3}=$ $\left(\tau_{1}-\tau_{3}\right), \xi_{4}=\left(-\tau_{2}+\tau_{4}\right)$ :

$$
\begin{aligned}
I_{4}= & \int_{0}^{\infty} d \xi_{1} e^{-4 \varpi_{\nu} \xi_{1}} I_{3}\left(\xi_{1}\right), \\
I_{3}\left(\xi_{1}\right) \equiv & \int_{-2 \xi_{1}}^{2 \xi_{1}} d \xi_{2} e^{-2 i n_{F} \xi_{2}} \int_{-\left(2 \xi_{1}+\xi_{2}\right)}^{2 \xi_{1}+\xi_{2}} \times \\
& d \xi_{3} \int_{-\left(2 \xi_{1}-\xi_{2}\right)}^{2 \xi_{1}-\xi_{2}} d \xi_{4} \frac{\beta(\gamma)}{\alpha_{1}+\alpha_{2}+\alpha_{3}+\alpha_{4}}
\end{aligned}
$$

The distribution function $I_{3}\left(\xi_{1}\right)$, has been calculated numerically for different integer values of $n_{F}$. Selecting integer values of $n_{F}$ pins the SC free energy at maxima of its magnetic quantum oscillations, allowing to determine their amplitude for any given harmonic in the dHvA frequency $F=H n_{F}$. The result for $I_{3}\left(\xi_{1}\right)$ is shown in Fig.1. It appears as a series of sharp peaks located around the points $\xi_{1}^{k}=\frac{\pi}{2} k$ with $k=0,1, .$. , having monotonically increasing intensity with increasing order $k$. The maximum positions of the peaks are slightly shifted with respect to $\frac{\pi}{2} k$ toward larger values due to the $\xi_{1}$-dependence of the $\xi_{3}, \xi_{4}$-integrals. The peaks' height is found to increase with increasing harmonic order $k$ as $k^{2}$, but the number of significantly contributing peaks is limited by the thermal damping factor $e^{-4 \varpi_{\nu} \xi_{1}}$. A simple estimation shows that for $2 \pi^{2} k_{B} T \gtrsim 4 \hbar \omega_{c}$ the contribution of the second harmonic does not exceed $10 \%$ of the first harmonic where the 3rd harmonic contribution is less than $1 \%$. On the other hand at temperatures as low as $2 \pi^{2} k_{B} T \lesssim \hbar \omega_{c}$ many harmonics provide comparable contributions. In this low temperature limit, replacing summation over harmonics with integration one finds for an integer $n_{F}: k_{B} T \sum_{\nu} \int_{0}^{\infty} d \xi_{1} e^{-4 \varpi_{\nu} \xi_{1}} I_{3}\left(\xi_{1}\right) \rightarrow$ 


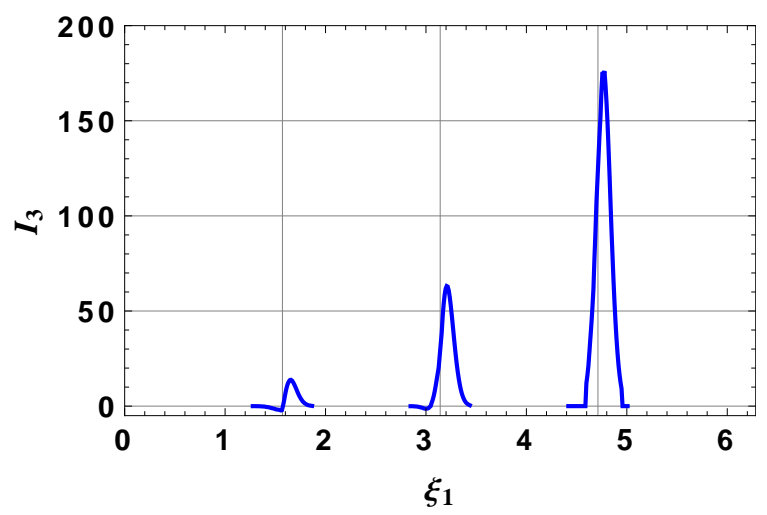

FIG. 1: The distribution function $I_{3}\left(\xi_{1}\right)$ given by Eq 27 for $n_{F}=32$. The peak near $\xi_{1}=0$ is too small to be observable in the considered scale .

$k_{B} T \sum_{\nu, k} k^{2} \exp \left[-\frac{\pi^{2} k_{B} T}{\hbar \omega_{c}}(2 \nu+1) k\right] \rightarrow \hbar \omega_{c}\left(\frac{\hbar \omega_{c}}{k_{B} T}\right)^{2}$. Therefore, the quartic term diverges as $\frac{1}{T^{2}}$ as $T \rightarrow 0$, due to the resonance pairing conditions ${ }^{2}$ characterizing the zero spin splitting situation considered here. Note, however, that the resulting divergence is weaker than that obtained in the local approximation ${ }^{2}$. In the latter the quartic term for an integer $n_{F}$ was found to be proportional to $k_{B} T \sum_{\nu} q_{\nu}^{2}$, with $q_{\nu}=\frac{e^{X_{\nu}}}{\cosh X_{\nu}+\cos 2 \pi\left(n_{F}+1 / 2\right)}$, and $X_{\nu}=\frac{2 \pi^{2} k_{B} T}{\hbar \omega_{c}}(2 \nu+1)$, with the following low temperature limit: $k_{B} T \sum_{\nu} q_{\nu}^{2} \rightarrow \frac{1}{T^{3}}$. Note also that the quadratic term, which is local in nature, is characterized by the low temperature limit: $k_{B} T \sum_{\nu} q_{\nu} \rightarrow \frac{1}{T}$.

For the sake of illustrating the novel (quantum) features of the theory we will focus here on the leading magnetic quantum oscillatory effect by considering the first harmonic of the thermodynamic potential in the $\mathrm{dHvA}$ frequency $F=H n_{F}$. This situation corresponds to the usual dHvA experimental conditions when higher harmonics are relatively small. Under these circumstances the main contribution to $I_{4}$ (see Eq (26) ) originates in the second peak at $\xi_{1} \simeq \pi / 2$, which is dominated by the integral over small intervals around $\xi_{2} \simeq \pm \pi$, and to lesser extent by all other values of $\xi_{2}$. The resulting integral over $\xi_{2}$ (with the integrand including $e^{-2 i n_{F} \xi_{2}}$, see Eq 27) in the small intervals near $\xi_{2} \simeq \pm \pi$ yield the dominant contribution to the first harmonic. As usual for the first harmonic one may restrict the thermal Matsubara summation to the single term $\nu=0$.

The integration over $\xi_{1}$ around the point $\xi_{1} \simeq \pi / 2$ has been performed for different integer values of $n_{F}$ un-

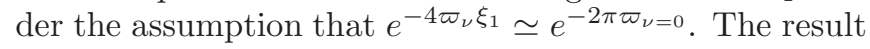
presented in Fig.2 (blue line) shows clearly the erratic oscillatory dependence on $n_{F}$ associated with the coupling to the vortex lattice.

The mean base line of this function, shown in the figure, corresponds to $I_{4}\left(\xi_{1} \simeq \pi / 2\right)$ calculated after replac-

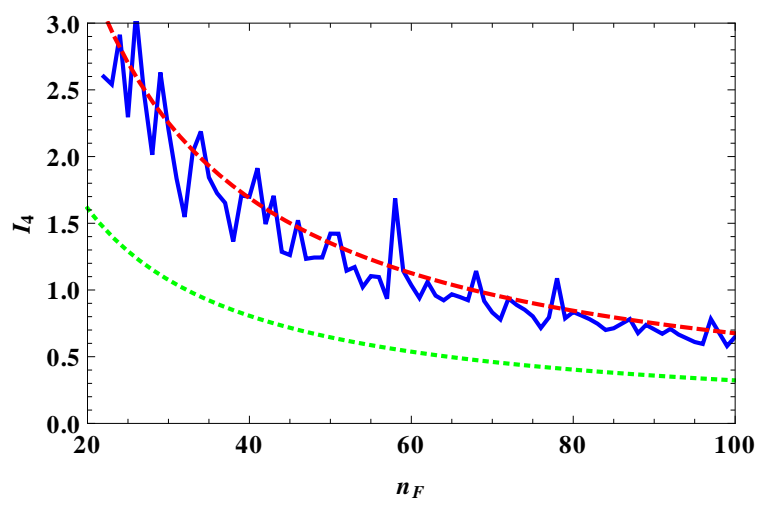

FIG. 2: The four-fold $\tau$ - integral, $I_{4}$, calculated at integer values of $n_{F}$ for a square vortex lattice (blue solid line). The red dashed line presents the harmonic part, obtained by using Eq. 28. Use of the first term in this expression (corresponding to the single pole at $\gamma=1$ ) in the calculation of $I_{4}$ yields the green dotted line.

$\operatorname{ing} \beta_{s q}(\gamma)$ with:

$$
\beta_{s q}^{(h)}(\gamma) \equiv \frac{1}{1-\gamma}+\frac{1}{1+\gamma}
$$

The result is purely harmonic, as can be seen by expanding one of the denominators, e.g. $\left(\alpha_{1}+\alpha_{2}+\alpha_{3}+\alpha_{4}\right)(1-\gamma) \rightarrow 0$ near $\tau_{1}, \tau_{3} \rightarrow$ $0 ; \tau_{2}, \tau_{4} \rightarrow \pi$, in the small variables $\left(\widetilde{\tau}_{i} \ll 1\right)$, $\widetilde{\tau}_{1}=\tau_{1}, \widetilde{\tau}_{2} \rightarrow-\pi+\tau_{2}, \widetilde{\tau}_{3} \rightarrow \tau_{3}, \widetilde{\tau}_{4} \rightarrow-\pi+\tau_{4}$, up to second order and keeping only leading terms in each variable (see Eq 24). The pole contribution at $\xi_{2}\left(\xi_{2}=-\pi+\widetilde{\xi}_{2}\right)$ yields the first harmonic $e^{2 i n_{F} \pi} e^{-2 n_{F}\left(\widetilde{\xi}_{1}^{2}+\frac{1}{4} \widetilde{\xi}_{3}^{2}\right)}$, which is strongly localized around the origin along both directions $\xi_{1}$ and $\xi_{3}$ with a characteristic width $\sim \frac{1}{\sqrt{n_{F}}}$. The integral over $\xi_{4}$ is not local and it is restricted only by its integration limits $\pm\left(2 \xi_{1}-\xi_{2}\right) \simeq \pm 2 \pi$. The remaining local (Gaussian) behavior in the corresponding 2D subspace enables one to estimate the global dependence of $I_{4}^{(1 h)}\left(\xi_{1} \simeq \pi / 2\right)$ on $n_{F}$ as $I_{4}^{(1 h)}\left(\xi_{1} \simeq \pi / 2\right) \sim \frac{1}{n_{F}}$.

Fig. 2 also confirms the conclusion drawn in Sec.IIIB3 on the basis of an analytical consideration saying that umklapp scattering of electron pairs by the vortex lattice via large reciprocal lattice vectors across the entire fermi sphere diameter leads to erratic oscillatory dependence of the thermodynamic potential on $n_{F}=\frac{F}{H}$ about the base line envelope $\sim \frac{1}{n_{F}}$. The absence of similar Umklapp scattering effects in the leading, quadratic term in the order parameter expansion, and their expected increasingly enhanced appearances in higher order terms of this expansion indicate that the irregularity discussed above should appear pronounced far from the SC transition where the quartic and higher order terms become important.

The final result for the first harmonic of the SC thermodynamic potential, up to fourth order, can be written 


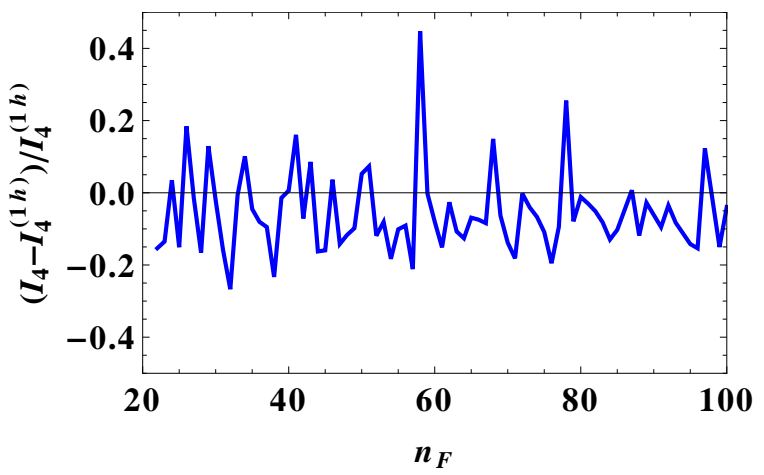

FIG. 3: The "erratic" function $w\left(n_{F}\right)$ showing the relative contribution to $I_{4}$ associated with the coherent scattering by the vortex lattice. Note that the negative jumps are due to paramagnetic distortions of the cyclotron orbits traversing through vortex core regions, whereas the positive jumps are associated with diamagnetic distortions.

in the form:

$$
\begin{aligned}
\Omega_{s c}^{(1 h)} / \Omega_{n}^{(1 h)} \simeq & 1-\frac{\pi^{3 / 2}}{\sqrt{n_{F}}}\left|\frac{\Delta_{0}}{\hbar \omega_{c}}\right|^{2} \\
& +\frac{1}{2} w_{0}\left(1+w\left(n_{F}\right)\right) \frac{\pi^{3}}{n_{F}}\left|\frac{\Delta_{0}}{\hbar \omega_{c}}\right|^{4}-\ldots
\end{aligned}
$$

where $w_{0} \simeq 1.1$ arises from the spatially uniform component of the SC order parameter, and is purely harmonic, whereas $w\left(n_{F}\right)$, shown in Fig.3, represents effects of umklapp scattering by the vortex lattice leading to deviations from the purely harmonic Fourier spectrum.

It is interesting to note that these "erratic" umklapp scattering processes can be viewed in real space as arising from the passages of paired electrons in cyclotron orbits (near the fermi energy) through vortex core regions, where the cyclotron orbit is strongly distorted by the pair-potential into small circular orbits around the vortex cores 2 . The resulting deviations from the normal state cyclotron orbit in a vortex core are paramagnetic or diamagnetic, depending on the electron energy relative to the Fermi surface, with the paramagnetic sectors leading to the sharp drops of the free energy shown in Fig.3, while the diamagnetic ones yielding the sharp rises seen there.

The existence of these erratic oscillations is due to the highly coherent cyclotron motions of the two pairs of electrons responsible for the singular terms $\gamma= \pm 1$ in Eq21 A scattering process of these electrons which can destroy this coherence should lead to removal of the singular behavior. Leaving to future publications the question of how such scattering processes can be implemented into the present theory (see the discussion in Sec.V), it is desirable to investigate the robustness of the quartic term $I_{4}$ with respect to smearing of the singularities at $\gamma= \pm 1$. This can be done by artificially shifting $1-\gamma$, and $1+\gamma$ in Eq[25] slightly away form their vanishing forms to $1+\sigma-\gamma$, and $1+\sigma+\gamma$ respectively, for small

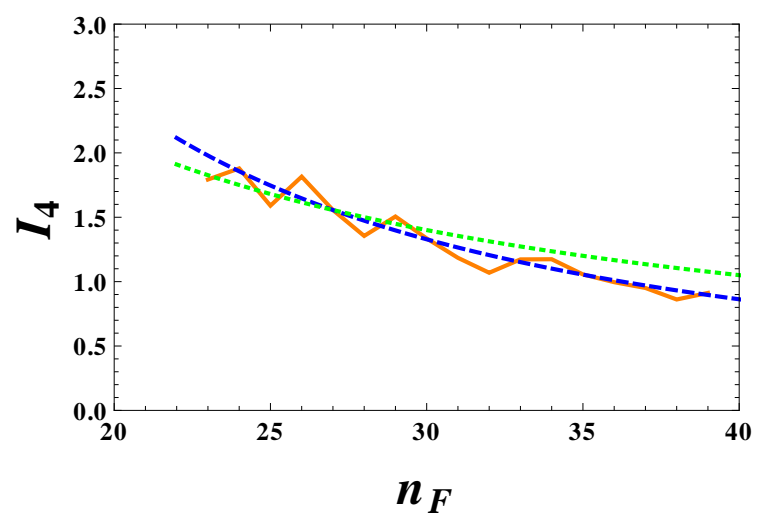

FIG. 4: $I_{4}$, calculated at integer values of $n_{F}$ for a square vortex lattice, as in Fig.2, in which the singularities at $\gamma=$ \pm 1 are removed (see text) with the regularization parameter $\sigma=.01$ (red solid line). The (blue) dashed line, which is proportional to $n_{F}^{-3 / 2}$, is the best fitting curve, whereas the (green) dotted curve follows a fitting formula proportional to $n_{F}^{-1}$.

values of $\sigma>0$, and repeating the calculation shown in Fig.2. The result for $\sigma=0.01$ is shown in Fig.4. In addition to the significant reduction of the overall magnitude and suppression of the (coherent-scattering) "erratic" oscillations, the $n_{F}$ dependence of the mean base line changes from $n_{F}^{-1}$ to $n_{F}^{-3 / 2}$, characterizing the local approximation of the GGL theory ${ }^{2}$.

\section{THE EFFECT OF VORTEX LATTICE DISORDER IN THE WHITE NOISE LIMIT}

Calculation of the influence of vortex-lattice disorder on the SC free energy in the magneto-quantum oscillations limit can be performed analytically in the white noise limit. Invoking the general expansion of the state function $\varphi_{0}(x, y)$ in terms of Landau orbitals wave functions, $\varphi_{0}(x, y)=e^{i x y} \sum_{n} c_{n} e^{i q_{n} x-\left(y+q_{n} / 2\right)^{2}}$, the structure factor takes the form:

$$
\begin{aligned}
\beta(\gamma)= & \frac{\sqrt{\pi}}{a_{x}} \frac{1}{\left(1-\gamma^{2}\right)^{1 / 2}} \frac{1}{N_{x}} \times \\
& \sum_{n s t} \exp \left[-\left(\frac{\pi}{a_{x}}\right)^{2}\left(\frac{1-\gamma}{1+\gamma} s^{2}+\frac{1+\gamma}{1-\gamma} t^{2}\right)\right] \times \\
& c_{n}^{*} c_{n+s+t}^{*} c_{n+s} c_{n+t}
\end{aligned}
$$

where the coefficients $\left\{c_{n}\right\}$ may be considered as random variables. Averaging the structure factor over realizations of these coefficients and exploiting the usual (Wick) 
decoupling:

$$
\begin{aligned}
& \langle\beta(\gamma)\rangle=\frac{\sqrt{\pi}}{a_{x}} \frac{1}{N_{x}\left(1-\gamma^{2}\right)^{1 / 2}} \\
& \sum_{n s t} \exp \left[-\left(\frac{\pi}{a_{x}}\right)^{2}\left(\frac{1-\gamma}{1+\gamma} s^{2}+\frac{1+\gamma}{1-\gamma} t^{2}\right)\right] \times \\
& {\left[\left\langle c_{n}^{*} c_{n+s}\right\rangle\left\langle c_{n+s+t}^{*} c_{n+t}\right\rangle+\left\langle c_{n+s+t}^{*} c_{n+s}\right\rangle\left\langle c_{n}^{*} c_{n+t}\right\rangle\right]}
\end{aligned}
$$

, in the white noise limit, i.e. $\left\langle c_{n}^{*} c_{n+s}\right\rangle\left\langle c_{n+s+t}^{*} c_{n+t}\right\rangle \rightarrow$ $\delta_{n, n+s} \delta_{n+s+t, n+t}=\delta_{s, 0}$, and $\left\langle c_{n+s+t}^{*} c_{n+s}\right\rangle\left\langle c_{n}^{*} c_{n+t}\right\rangle \rightarrow$ $\delta_{n+s+t, n+s} \delta_{n, n+t}=\delta_{t, 0}$, one finds:

$$
\langle\beta(\gamma)\rangle=\frac{\sqrt{\pi}}{a_{x}} \frac{1}{\left(1-\gamma^{2}\right)^{1 / 2}}\left[\begin{array}{c}
\sum_{s} e^{-\left(\frac{\pi}{a_{x}}\right)^{2}\left(\frac{1-\gamma}{1+\gamma}\right) s^{2}} \\
+\sum_{t} e^{-\left(\frac{\pi}{a_{x}}\right)^{2}\left(\frac{1+\gamma}{1-\gamma}\right) t^{2}}
\end{array}\right]
$$

The final step in the procedure leading to the white noise limit should be the replacement of the discrete summations in Eq 31 with integrations (e.g. by taking $a_{x} \rightarrow \infty$ there), resulting in the expression:

$$
\langle\beta(\gamma)\rangle \rightarrow \frac{1}{1-\gamma}+\frac{1}{1+\gamma}
$$

This is a rather surprising results since it is seen to be twice Eq 22, obtained for the forward scattering term. The latter (i.e. the $G=0$ term), which is usually associated with all incoherent scattering processes, is expected to be the sole survivor of an averaging over white-noise disorder, and as such to coincide with Eq 32

In this limiting case, only incoherent scattering processes by the vortex matter contribute to the SC thermodynamic potential, and the final result, up to fourth order, is purely harmonic, with the first harmonic given by:

$$
\left\langle\Omega_{s c}^{(1 h)}\right\rangle / \Omega_{n}^{(1 h)} \simeq 1-\frac{\pi^{3 / 2}}{\sqrt{n_{F}}}\left|\frac{\Delta_{0}}{\hbar \omega_{c}}\right|^{2}+\frac{1}{2} w_{0} \frac{\pi^{3}}{n_{F}}\left|\frac{\Delta_{0}}{\hbar \omega_{c}}\right|^{4}-\ldots
$$

i.e., very close to the well known Maki-Stephen expression ${ }^{14.15}$, as expanded to the same order in $\Delta_{0}$.

An interesting question arises here as to wether the white-noise average of higher order terms in the orderparameter expansion presented in this paper also agree with the self-consistent Born approximation (SCBA) inherent to the Maki-Stephen approach ${ }^{2}$. In particular, possible destruction of the highly coherent motions of the electron pairs responsible for the singular contributions to the quartic term $I_{4}$ by an infinite subset of diagrams which are topologically distinct from the quartic diagram, might lead to significant deviations from the SCBA.

\section{CONCLUSION AND DISCUSSION}

A novel Green's function representation is exploited in this paper for a microscopic derivation of the Ginzburg-
Landau theory of strongly type superconductivity at high magnetic fields. An exact analytical expression for the quartic term in the corresponding order parameter expansion, having a physically transparent form, is presented. The resulting expression reveals singular nonlocal contributions to the SC thermodynamic potential, associated with highly coherent cyclotron motions of the paired electrons near the Fermi surface, which are strongly coupled to the vortex lattice. The dominant contributions to the SC free energy, arise from incoherent scattering by the spatially averaged pair-potential, which is purely harmonic in the dHvA frequency. However, coherent scatterings by the ordered vortex lattice generate, at low temperatures, erratically oscillating (i.e. paramagnetic-diamagnetic) contribution to the SC free energy as a function of the magnetic field, associated with sharp distortions of the large quasi-particle cyclotron orbits on the Fermi surface traversing through vortex core regions. Vortex lattice disorder, which tends to suppress this oscillatory component, is found to simplify considerably the calculation allowing analytical evaluation of higher order terms in the order-parameter expansion. However, it can be shown that the infinite subset of diagrams constituting the standard, self consistent Born approximation $(\mathrm{SCBA})^{15}$, exploited in the white noise limit of the discorded vortex system ${ }^{2}$, have the same type of singular points as that found in our calculation of the quartic term. It would be therefore very interesting to search for, and then evaluate subsets of diagrams, topologically distinct from those appearing in the SCBA, which might, after resummation, destroy the highly coherent cyclotron motions responsible for the above singularities. Physically speaking, it is expected that the effect of impurity-scattering on the paired electrons, as calculated beyond the relaxation time approximation, could destroy this coherence. Whether or not the robustness of this type of singularities with respect to scattering of quasi particles by a disordered vortex matter is destroyed by going beyond the SCBA is a crucial question in our understanding of the vortex lattice disorder on the $\mathrm{dHvA}$ oscillations in the SC state $\frac{16}{}$.

\section{Acknowledgments}

This research was supported by the Israel Science Foundation, by Posnansky Research fund in superconductivity, and by EuroMagNET under the EU contract No. 228043.

\section{Appendix A}

Similar to the calculation of the quadratic term, it is convenient to introduce the following, center of mass and 
relative coordinates:

$$
\begin{aligned}
& \mathbf{R}=\frac{1}{4}\left(\mathbf{r}_{1}+\mathbf{r}_{\mathbf{2}}+\mathbf{r}_{\mathbf{3}}+\mathbf{r}_{4}\right) \\
& \mathbf{Q}=\frac{1}{2}\left(\mathbf{r}_{1}-\mathbf{r}_{\mathbf{2}}+\mathbf{r}_{3}-\mathbf{r}_{4}\right)=\frac{1}{4}\left(\rho_{1}-\rho_{2}+\rho_{3}-\rho_{4}\right) \\
& \mathbf{D}=\frac{1}{2}\left(\mathbf{r}_{1}-\mathbf{r}_{2}-\mathbf{r}_{3}+\mathbf{r}_{4}\right)=\frac{1}{2}\left(\rho_{4}-\rho_{2}\right) \\
& \mathbf{P}=\frac{1}{2}\left(\mathbf{r}_{1}+\mathbf{r}_{\mathbf{2}}-\mathbf{r}_{3}-\mathbf{r}_{4}\right)=\frac{1}{2}\left(\rho_{1}-\rho_{3}\right)
\end{aligned}
$$

where $\rho_{i}=\mathbf{r}_{\mathbf{i}}-\mathbf{r}_{\mathbf{i}-\mathbf{1}}$. This transformation can be written in the matrix form: $\boldsymbol{X}=M * \mathbf{r}$, where $\boldsymbol{X} \equiv$ $\{\mathbf{R}, \mathbf{Q}, \mathbf{D}, \mathbf{P}\}$ are four $2 \mathrm{D}$ vectors and $M$ is a $4 \times 4$ matrix with $|\operatorname{det} M|=1 / 2$.

All ingredients of the quartic term, which depend on the electronic spatial coordinates, i.e.:

$$
\begin{aligned}
\Omega_{4}= & \Omega_{4}^{(0)} \int d^{2}\{\mathbf{r}\} \widetilde{\Gamma}_{4}(\{\mathbf{r}\}) \widetilde{K}_{4}(\{\mathbf{r}\}), \\
\Omega_{4}^{(0)}= & \frac{2 \pi}{a_{x}^{2}} \frac{1}{(2 \pi)^{4}} k_{B} T a_{H}^{2}\left|\frac{\Delta_{0}}{\hbar \omega_{c}}\right|^{4} \\
\widetilde{K}_{4}(\{\mathbf{r}\})= & \int_{0}^{\infty} d \tau_{1} d \tau_{2} d \tau_{3} d \tau_{4} e^{-i\left(\tau_{1}-\tau_{2}+\tau_{3}-\tau_{4}\right) n_{F}-\varpi_{\nu}\left(\tau_{1}+\tau_{2}+\tau_{3}+\tau_{4}\right)} \times \\
& \frac{1}{\alpha_{1} \alpha_{2} \alpha_{3} \alpha_{4}} \exp \left[-\left(\mu_{1} \rho_{1}^{2}+\mu_{2} \rho_{2}^{2}+\mu_{3} \rho_{3}^{2}+\mu_{4} \rho_{4}^{2}\right)\right] \\
\widetilde{\Gamma}_{4}(\{\mathbf{r}\})= & g^{*}\left(\mathbf{r}_{1}, \mathbf{r}_{2}\right) g\left(\mathbf{r}_{2}, \mathbf{r}_{3}\right) g^{*}\left(\mathbf{r}_{3}, \mathbf{r}_{4}\right) g\left(\mathbf{r}_{4}, \mathbf{r}_{1}\right) \varphi_{0}\left(\mathbf{r}_{1}\right) \varphi_{0}^{*}\left(\mathbf{r}_{2}\right) \varphi_{0}\left(\mathbf{r}_{3}\right) \varphi_{0}^{*}\left(\mathbf{r}_{4}\right)
\end{aligned}
$$

will be rewritten now in terms of the new coordinates. Let us start with the gauge factors, $g^{\star}\left(\mathbf{r}_{1}, \mathbf{r}_{\mathbf{2}}\right) g\left(\mathbf{r}_{\mathbf{2}}, \mathbf{r}_{\mathbf{3}}\right) g^{\star}\left(\mathbf{r}_{\mathbf{3}}, \mathbf{r}_{\mathbf{4}}\right) g\left(\mathbf{r}_{\mathbf{4}}, \mathbf{r}_{\mathbf{1}}\right)=e^{\eta_{g}}$, where:

$$
\eta_{g}=\frac{i}{2}\left(\left[\mathbf{r}_{\mathbf{1}} \times \mathbf{r}_{\mathbf{2}}\right]-\left[\mathbf{r}_{\mathbf{2}} \times \mathbf{r}_{\mathbf{3}}\right]+\left[\mathbf{r}_{\mathbf{3}} \times \mathbf{r}_{\mathbf{4}}\right]-\left[\mathbf{r}_{\mathbf{4}} \times \mathbf{r}_{\mathbf{1}}\right]\right)=2 i\left(Q_{x} R_{y}-Q_{y} R_{x}\right)
$$

which depends only on the vectors $\mathbf{R}, \mathbf{Q}$.

The product of the four Landau orbitals, labeled by $n_{1}=n+s+t, n_{2}=n+s, n_{3}=n$, and $n_{4}=n+t$, is given by the following expression:

$$
\varphi_{0 n_{1}}\left(\mathbf{r}_{1}\right) \varphi_{0 n 2}^{*}\left(\mathbf{r}_{2}\right) \varphi_{0 n_{3}}\left(\mathbf{r}_{3}\right) \varphi_{0 n_{4}}^{*}\left(\mathbf{r}_{4}\right)=\exp \left[\eta_{\Delta}^{(m)}+\eta_{\Delta}^{(s q)}+\eta_{\Delta}^{(l i n)}+\eta_{\Delta}^{(0)}\right]
$$

with:

$$
\begin{aligned}
\eta_{\Delta}^{(m)} & =i \sum_{j} \varepsilon_{j} x_{j} y_{j}=i\left[2 Q_{x} R_{y}+2 Q_{y} R_{x}+\left(D_{x} P_{y}+P_{x} D_{y}\right)\right] \\
\eta_{\Delta}^{(s q)} & =-\sum_{j} y_{j}^{2}=-\left(Q_{y}^{2}+4 R_{y}^{2}+\left(D_{y}^{2}+P_{y}^{2}\right)\right) \\
\eta_{\Delta}^{(l i n)} & =\sum_{j}\left(i \varepsilon_{j} q_{n_{j}} x_{j}-q_{n_{j}} y_{j}\right) \\
& =i \sum_{j}\left(\varepsilon_{j} n_{j}\right) R_{x}+i q_{0} \frac{1}{2}\left(Q_{x} N_{4}-2 D_{x} t-2 P_{x} s\right)-q_{0}\left(R_{y} N_{4}-D_{y} s-P_{y} t\right) \\
\eta_{\Delta}^{(0)} & =-\frac{1}{4} q_{0}^{2} \sum_{j} n_{j}^{2}=-\frac{1}{2} q_{0}^{2}\left[2 n^{2}+2 n s+2 n t+s t+s^{2}+t^{2}\right]
\end{aligned}
$$

and: $N_{4} \equiv \sum n_{j}=4 n+2 s+2 t$.

The last factor, $\widetilde{K}_{4}\left(\{\mathbf{r}\} \sim \exp \left(\eta_{G}\right)\right.$, arising from the transitional invariant parts of the Green functions, is independent of the center of mass coordinates:

$$
\eta_{G}=-\sum \mu_{j} \rho_{j}^{2}=-\left[\begin{array}{c}
\left(\mu_{1}+\mu_{2}+\mu_{3}+\mu_{4}\right) Q^{2}+2\left(\mu_{2}-\mu_{4}\right)(D Q)+ \\
2\left(\mu_{1}-\mu_{3}\right)(P Q)+\left(\mu_{2}+\mu_{4}\right) D^{2}+\left(\mu_{1}+\mu_{3}\right) P^{2}
\end{array}\right] .
$$


The simplest integration to carry out, over $R_{x}$, yields a non-vanishing result only if $\sum\left(\varepsilon_{j} n_{j}\right)=0$, justifying the parametrization of $n_{j}$ chosen above. In this case $\int d R_{x}=L_{x}=a_{x} N_{x}$. Next, the $R_{y}$-integration,

$$
\int \exp \left[4 i Q_{x} R_{y}-4 R_{y}^{2}-q_{0} N R_{y}\right] d R_{y}=\frac{1}{2} \sqrt{\pi} \exp \left[\frac{1}{16} q_{0}^{2} N^{2}-\frac{1}{2} i q_{0} N Q_{x}-Q_{x}^{2}\right]
$$

leads to a space independent correction, $\frac{1}{16} q_{0}^{2} N^{2}$, which removes the $n$-dependence of $\eta_{\Delta}^{(0)}: \eta_{\Delta}^{(0)}+\frac{1}{16} q_{0}^{2} N^{2}=$ $-\frac{1}{4} q_{0}^{2}\left(s^{2}+t^{2}\right)$. As a result, summation over Landau orbitals is trivially done, yielding the total number of orbitals $\sum_{n} 1=N_{y}$.

Combining the $Q$-dependent terms the corresponding integral is:

$$
\int d^{2} Q \exp \left[-\beta_{0}\left[Q^{2}+2 \beta_{24}(D Q)+2 \beta_{13}(P Q)\right]\right]=\frac{\pi}{\beta_{0}} \exp \left[\beta_{0}\left(\beta_{24}^{2} D^{2}+\beta_{13}^{2} P^{2}+2 \beta_{24} \beta_{13}(D P)\right)\right]
$$

where

$$
\beta_{0}=\mu_{1}+\mu_{2}+\mu_{3}+\mu_{4}+1, \beta_{24}=\frac{\mu_{2}-\mu_{4}}{\beta_{0}}, \beta_{13}=\frac{\left(\mu_{1}-\mu_{3}\right)}{\beta_{0}}
$$

The most complicated analytical part of the calculation, the $D P$-integrations, is now done by introducing the $4 \mathrm{D}$ vectors:

$$
Z=\left\{D_{x}, D_{y}, P_{x}, P_{y}\right\}, L=q_{0}\{-i t, s,-i s, t\}
$$

and the $\tau$ dependent $4 \times 4$ matrix:

$$
U=\left(\begin{array}{cccc}
\left(\mu_{2}+\mu_{4}\right)-\beta \beta_{24}^{2} & 0 & -\beta \beta_{24} \beta_{13} & -i / 2 \\
0 & \left(\mu_{2}+\mu_{4}\right)-\beta \beta_{24}^{2}+1 & -i / 2 & -\beta \beta_{24} \beta_{13} \\
-\beta \beta_{24} \beta_{13} & -i / 2 & \left(\mu_{1}+\mu_{3}\right)-\beta \beta_{13}^{2} & 0 \\
-i / 2 & -\beta \beta_{24} \beta_{13} & 0 & \left(\mu_{1}+\mu_{3}\right)-\beta \beta_{13}^{2}+1
\end{array}\right)
$$

and then performing the resulting Gaussian integrations to have:

$$
\int d^{4} Z \exp \left[-Z^{T} U Z+L Z\right]=\frac{\pi^{2}}{\sqrt{\operatorname{det} U}} \exp \left[L^{T} U^{-1} L\right]
$$

* e-mail:maniv@tx.technion.ac.il

1 M. Rasolt and Z. Tesanovic, Rev. Mod. Phys. 64, 709 (1992).

2 T. Maniv, V. Zhuravlev, I. D. Vagner, and P. Wyder, Rev. Mod. Phys. 73, 867 (2001).

3 T. J. B. M. Janssen, C. Haworth, S. M. Hayden, P. Meeson, M. Springford, and A. Wasserman, Phys. Rev. B 57, 11698 (1998).

4 R. Corcoran, N. Harrison, S. M. Hayden, P. Meeson, M. Springford, and P. J. van der Wel, Phys. Rev. Lett. 72, 701 (1994).

5 T. Terashima, C. Haworth, H. Takeya, S. Uji, H. Aoki, and K. Kadowaki, Phys. Rev. B 56, 5120 (1997).

6 T. Maniv, V. Zhuravlev, J. Wosnitza, O. Ignatchik, B. Bergk, and P. C. Canfield, Phys. Rev. B 73, 134521 (2006).

7 T. Isshiki, N. Kimura, H. Aoki, T. Terashima, S. Uji, K. Yamauchi, H. Harima, D. Jaiswal-Nagar, S. Ramakrish- nan, and A. K. Grover, Phys. Rev. B 78, 134528 (2008).

8 B. Bergk and J. Wosnitza, Low Temp. Phys. 35, 687 (2009).

9 S. Dukan and Z. Tesanovic, Phys. Rev. B 49, 13017 (1994)

10 S. Dukan and Z. Tesanovic, Phys. Rev. Lett. 74, 2311 (1995).

11 M. R. Norman, A.H. MacDonald, and H. Akera, Phys. Rev. B 51, 5927 (1995);

12 M. R. Norman, A.H. MacDonald, Phys. Rev. B 54, 4239 (1996).

13 K. Yasui and T. Kita, Phys. Rev. B 66, 184516 (2002).

14 K. Maki, Phys. Rev. B 44, 2861 (1991).

15 M.J. Stephen, Phys. Rev. B 45, 5481 (1992).

16 A. Maniv, T. Maniv, V. Zhuravlev, B. Bergk, J. Wosnitza, A. Kohler, G. Behr, P.C. Canfield, and J.E. Sonier, Phys. Rev. B 83, 104505 (2011). 